\section{Excesso de peso em crianças indígenas Xukuru do Ororubá, Pernambuco, Brasil: magnitude e fatores associados}

\author{
Excess weight in Xukuru indigenous children in \\ Ororubá, Pernambuco State, Brazil: magnitude \\ and associated factors
}

Exceso de peso en niños indígenas Xukuru do Ororubá, Pernambuco, Brasil: magnitud y factores asociados
Thatiana Regina Fávaro 1

Aline Alves Ferreira 2

Geraldo Marcelo da Cunha 3

Carlos E. A. Coimbra Jr. ${ }^{3}$

\title{
Resumo
}

Os poucos estudos que abordam o tema de saúde e nutrição em indígenas da macrorregião Nordeste do Brasil sinalizaram situações diferenciadas em alguns aspectos, quando comparados principalmente à realidade de indígenas do Norte e Centro-oeste. Este trabalho objetivou estimar a magnitude das prevalências e risco de sobrepeso e excesso de peso em crianças menores de dez anos da etnia Xukuru do Ororubá, Estado de Pernambuco, e avaliar os fatores socioeconômicos e demográficos potencialmente associados a estes agravos. Estudo transversal, no qual realizou-se uma análise da associação entre as variáveis de desfecho, peso adequado, excesso de peso (sobrepeso e obesidade) e risco de sobrepeso, de acordo com os índices e pontos de corte da Organização Mundial da Saúde para crianças e as variáveis explicativas, utilizando-se regressão logística multinomial. A prevalência de excesso de peso foi de 7,7\% $e$ de risco de sobrepeso de 24,2\%. As chances de risco de sobrepeso e de excesso de peso são maiores em crianças < 2 anos, e filhos de mães obesas apresentam maior chance de excesso de peso. A prevalência de risco de sobrepeso foi 97\% maior quando comparada com os domicílios sem renda fixa. Os achados sugerem que os Xukuru estão atravessando um acelerado processo de transição nutricional, com uma situação paradoxal a que outros povos indígenas no Brasil estão expostos.

Saúde de Populações Indígenas; Índios Sul-americanos; Estado Nutricional; Obesidade Pediátrica

\author{
Correspondência \\ T. R. Fávaro \\ Faculdade de Nutrição, Universidade Federal de Alagoas. \\ Av. Lourival Melo Mota s/n, Maceió, AL 57072-900, Brasil. \\ thatifavaro@gmail.com \\ 1 Faculdade de Nutrição, Universidade Federal de Alagoas, \\ Maceió, Brasil. \\ 2 Instituto de Nutrição Josué de Castro, Universidade Federal do \\ Rio de Janeiro, Rio de Janeiro, Brasil. \\ 3 Escola Nacional de Saúde Pública Sergio Arouca, Fundação \\ Oswaldo Cruz, Rio de Janeiro, Brasil.
}




\section{Introdução}

A Organização Mundial da Saúde (OMS) é categórica ao afirmar que o excesso de peso e a obesidade são agravos relevantes na qualidade de vida das populações, e estão relacionados às principais causas de morbimortalidade em praticamente todas as faixas de idade. Em 2016, 41 milhões de crianças menores de 5 anos e 340 milhões de crianças e adolescentes com idades entre 5 e 19 anos estavam acima do peso ou obesas 1 .

O excesso de peso e a obesidade infantil são agravos de etiologia complexa, geralmente associados a variáveis comportamentais, socioeconômicas e demográficas 1,2,3. Crianças obesas ou com excesso de peso têm maior probabilidade de desenvolver doenças crônicas não transmissíveis (DCNTs) precocemente, além de possuírem maiores chances de terem outras morbidades 1,4.

No Brasil, inquéritos nacionais têm ressaltado a modificação do perfil nutricional das crianças brasileiras, principalmente nas últimas três décadas, com a diminuição acentuada de todas as formas de desnutrição e aumento na incidência de excesso de peso em crianças maiores de 5 anos, independentemente da macrorregião ou renda ${ }^{5}$. Esse panorama consolidado por inquéritos da população no país não inclui alguns segmentos populacionais vulneráveis, incluindo os indígenas.

Resultados de trabalhos realizados com crianças indígenas no Brasil nos últimos 20 anos, compactuam que os principais problemas em relação ao estado nutricional infantil estão relacionados a carências nutricionais, como anemia, baixo peso e déficit de crescimento 6,7,8,9. A única pesquisa nacional (I Inquérito Nacional de Saúde e Nutrição dos Povos Indígenas) que avaliou especificamente essa população identificou que a prevalência de déficit de crescimento atingia cerca de um quarto das crianças indígenas do país, sendo o Nordeste a região com os menores valores percentuais. Por outro lado, os resultados desse Inquérito apontaram que $15,1 \%$ das crianças indígenas $<5$ anos da Região Nordeste apresentaram risco de excesso de peso 10.

De maneira geral, os poucos estudos que abordam aspectos relacionados à saúde e nutrição de indígenas da macrorregião Nordeste do Brasil, revelam situações distintas em muitos aspectos quando comparados principalmente à realidade do Norte e Centro-oeste 8,11,12,13. Os Xukuru do Ororubá constituem um dos maiores contingentes populacionais indígenas do Nordeste e o maior do Estado de Pernambuco. Totalizam aproximadamente 8 mil indivíduos residentes em 25 aldeias na Terra Indígena Xukuru ${ }^{14}$. O excesso de peso entre os adultos Xukuru do Ororubá já foi sinalizado em altas prevalências destacando, assim como outros povos indígenas, um acelerado e complexo processo de transição alimentar e nutricional 15.

O objetivo deste estudo foi estimar a magnitude das prevalências e risco de sobrepeso e excesso de peso em crianças menores de dez anos da etnia Xukuru do Ororubá, de Pernambuco, e avaliar os fatores socioeconômicos e demográficos potencialmente associados a estes agravos.

\section{Materiais e métodos}

Este estudo, de corte transversal e base populacional, foi realizado na Terra Indígena Xukuru, Município de Pesqueira, Pernambuco. A população-alvo deste trabalho consistiu em crianças $<10$ anos.

O presente estudo compôs uma ampla investigação sobre o perfil de saúde e nutrição do povo Xukuru do Ororubá, que envolveu todas as faixas de idade e avaliou uma série de desfechos em saúde. Desse modo, a amostra inicial foi calculada para representar o total de crianças com idades entre $6 \mathrm{e}$ 59 meses, considerando-se a prevalência de anemia de $40 \%$, nível de confiança de 0,05 e com precisão de 0,95 . A escolha por estimar a amostra com base de prevalência de anemia se deve por ser o agravo mais prevalente dentre os investigados na pesquisa maior. Crianças na faixa de idade objeto deste estudo totalizavam 1.692, e para atender especificamente os objetivos desta investigação a amostra foi recalculada considerando-se a prevalência estimada de excesso de peso em crianças menores de 10 anos em $20 \%$, precisão de 0,99 e nível de confiança de 0,05 . Dessa maneira, deveriam compor a amostra 425 crianças. $O$ cálculo amostral inicialmente realizado resultou na inclusão de 454 crianças menores de 10 anos, sendo assim, superior ao necessário.

Com base na informação de que existiam em torno de 2.100 domicílios em toda a Terra Indígena e considerando os domicílios como unidade de observação planejou-se amostrar um terço do total, ou 
seja, 700 domicílios. A seleção dos domicílios ocorreu de forma aleatória sistemática, por meio de sorteio entre 1 e k, em que k seria o número total de domicílios. Vejam mais detalhes em Fávaro et al. 15.

A pesquisa de campo foi realizada nos meses de janeiro e março de 2010. As informações socioeconômicas e demográficas foram obtidas em janeiro e fevereiro, valendo-se do diagnóstico sociodemográfico participativo dos Xukuru do Ororubá 16, no qual os entrevistadores foram agentes indígenas de saúde e de saneamento que trabalhavam na terra indígena, treinados e continuamente supervisionados durante a pesquisa de campo. Os dados antropométricos e as demais informações foram coletados por uma equipe de 16 entrevistadores devidamente treinados e supervisionados, seguindo o protocolo de Lohman et al. ${ }^{17}$. Todos os instrumentos de coleta de dados foram verificados e possíveis inconsistências eram imediatamente checadas.

\section{Variáveis investigadas}

As variáveis de desfecho investigadas foram: peso adequado, risco de sobrepeso e excesso de peso. Para tal, foram coletadas as medidas de peso corporal e altura, e calculado o índice de massa corporal (IMC). Para a aferição desses dados foram utilizadas balança digital portátil marca Seca (modelo 872, Hamburgo, Alemanha) e estadiômetro desmontável marca Alturexata (Belo Horizonte, Brasil). Para garantir a acurácia da medida da altura, estas foram tomadas em duplicata, sendo usada a média das medidas para as análises.

Em associação com a idade, foi construído o índice IMC-para-idade (IMC/I), cujos valores foram transformados em escores z com base nas referências da OMS 18,19, com o auxílio do software Anthro (http://www.who.int/childgrowth/software/en/). O estado nutricional das crianças foi classificado da seguinte forma, por valores de escores $\mathrm{z}$ : baixo peso $(\leq-2,00)$; peso adequado $(>-2,00$ e $<1,00)$; risco de sobrepeso $(\geq 1,00$ e $<2,00)$; sobrepeso $(\geq 2,00$ e $<3,00)$ e obesidade $(\geq 3,00)$. As categorias de sobrepeso e obesidade foram unidas para a criação da variável "excesso de peso". As crianças classificadas em baixo peso foram excluídas das análises, uma vez que não compõem os objetivos deste estudo.

\section{- Variáveis independentes}

As variáveis socioeconômicas e demográficas usadas foram: renda domiciliar per capita; renda fixa; índice de status econômico (ISE); tipo de domicílio e saneamento; número de moradores no domicílio (1-4; $5-8 ;>8$ ). A variável renda per capita domiciliar foi definida baseando-se na soma dos valores monetários recebidos pelos moradores do domicílio com mais de dez anos de idade (incluindo renda de trabalho assalariado, pensões, aposentadoria, programas de transferência de renda e venda de produtos de artesanato e agropecuários), dividida pelo número de moradores do domicílio. Com base no valor do salário mínimo à época da coleta dos dados, as rendas per capita foram organizadas em quatro categorias $(<$ 1 salário mínimo; $\geq 1$ e $<2 ; \geq 2$ e $<3 ; \geq 3$ ). Domicílio com renda fixa foi aquele com o morador recebendo aposentadoria, pensão ou com trabalho assalariado no mês anterior à entrevista. O ISE do domicílio foi obtido valendo-se da soma (em Reais) dos valores (estimados com base nos preços no comércio regional) dos seguintes bens de consumo presentes nos domicílios: fogão a gás, geladeira, máquina de lavar roupa, ar condicionado, televisão, antena parabólica, vídeo cassete ou DVD, aparelho de som com CD, microcomputador, telefone celular e bicicleta. Foi calculada a razão $\frac{S_{x}}{\max \left(S_{x}\right)}$, na qual $S_{x}$ correspondeu à soma dos valores dos bens de um dado domicílio e $\max \left(S_{x}\right)$ ao maior ISE observado em todos os domicílios. Esse indicador foi estratificado em três categorias com base nos tercis da distribuição.

As variáveis referentes ao domicílio foram categorizadas em dois tipos. Domicílios do tipo A foram os que apresentaram paredes de alvenaria ou tijolo, ou de madeira apropriada e piso de cerâmica ou cimento, e telha de barro ou laje. Habitações com paredes de madeira reaproveitada ou taipa/ barro, com piso de terra ou outro material e cobertura de lona, plástico ou outro material foram denominadas tipo B. As condições de saneamento foram classificadas em três categorias: (1) "Adequado": domicílios com escoadouros ligados à rede geral ou fossa séptica, servidos de água proveniente de rede geral de abastecimento ou da Fundação Nacional de Saúde (Funasa) e lixo coletado direta ou indiretamente por serviço de limpeza pública; (2) "Semiadequado": domicílios com, pelo menos, um dos serviços de abastecimento de água, esgoto ou lixo classificados como adequado; (3) "Inadequado": domicílios com escoadouro ligado à fossa rudimentar, vala, rio ou açude e outro escoadouro; 
servidos de água proveniente de poço ou nascente (ou outra forma), com lixo queimado ou enterrado, ou jogado em terreno baldio foram classificados como "inadequados".

Ainda foram obtidas informações sobre a escolaridade materna ( $\leq 4 ; 5-8 ; \geq 9$ anos completos) e sobre o trabalho materno fora de casa (sim; não).

As variáveis materno-infantis foram categorizadas da seguinte forma: idade materna $(<20 ; \geq 20 \mathrm{e}$ $<35$; $\geq 35)$; estatura materna (<150cm; $\geq 150)$; IMC da mãe $\left(<25 \mathrm{~kg} / \mathrm{m}^{2} ; \geq 25\right.$ e $\left.<30 ; \geq 30\right)$; número de filhos ( $\leq 3$; > 3); situação conjugal (com companheiro/a; sem companheiro/a); idade da criança $(<12$ meses; $\geq 12$ e < 24; $\geq 24$ e < 36; $\geq 36$ e < 48; $\geq 48)$; peso ao nascer $(<2.500$ g; $\geq 2.500$ e $<3.000 ; \geq 3.000$ e $<4.000$; $\geq 4.000$ ); sexo (masculino; feminino).

\section{Análise de dados e permissões éticas}

Inicialmente, foram calculadas as prevalências de baixo peso, peso adequado, risco de sobrepeso e excesso de peso para as categorias de cada variável independente. A análise dos efeitos das variáveis foi realizada em duas etapas por meio de modelos de regressão logística multinomial, tendo como desfecho o peso, classificado como peso adequado, risco de sobrepeso e excesso de peso. Na primeira etapa, utilizaram-se modelos não ajustados. As variáveis estatisticamente significativas no nível de $20 \%$ foram selecionadas. Na segunda, o modelo foi ajustado por inclusão de variáveis realizadas passo a passo, sendo incluídas, ao final, as variáveis estatisticamente significantes $(\mathrm{p}<0,05)$. Os modelos foram comparados por meio do critério de informação de Akaike (AIC), que considera um trade-off entre a qualidade do ajuste e o número de parâmetros no modelo. Após a inclusão simultânea de todos os efeitos principais foram testadas as interações plausíveis.

Foram usados os programas estatísticos IBM SPSS (https://www.ibm.com/), versão 21.0, e bibliotecas epicalc e sandwich do software R (http://www.r-project.org), versão 3.4.3.

O trabalho foi aprovado pelo Comitê de Ética em Pesquisa do Centro de Pesquisas Aggeu Magalhães, da Fundação Oswaldo Cruz, e pela Comissão Nacional de Ética em Pesquisa (CONEP, parecer no 592/09). Os investigados foram informados quanto à participação voluntária no estudo, sendo esclarecidos os objetivos e processos da pesquisa. O Termo de Consentimento Livre e Esclarecido foi assinado pelas lideranças da Terra Indígena Xukuru.

\section{Resultados}

Foram investigadas 454 crianças, sendo que para as análises foram excluídas quatro crianças com baixo peso (0,9\%). Não foram registradas recusas dos responsáveis na participação das crianças no estudo. A prevalência de excesso de peso (sobrepeso e obesidade) foi de 7,7\% $(\mathrm{n}=18)$ e de risco de sobrepeso foi de $24,2 \%$ ( $n=56$ ) (dados não apresentados).

A distribuição da população investigada segundo as variáveis independentes e o desfecho é apresentada na Tabela 1. A prevalência de excesso de peso foi maior para as meninas e em crianças com idade $\geq 2$ e $<5$ anos. Os maiores valores de excesso de peso também foram encontrados nas crianças cuja mãe tinha mais de 35 anos e apresentaram o IMC acima de $30 \mathrm{~kg} / \mathrm{m}^{2}$, e também para aquelas que viviam em domicílios com renda per capita $\geq 3$ salários mínimos e com saneamento adequado.

$\mathrm{Na}$ análise bruta da regressão logística multinominal (Tabela 2) destacaram-se alguns resultados. As chances de risco de sobrepeso e de excesso de peso são maiores em crianças com menos de dois anos de idade. Filhos de mães obesas apresentaram maiores chances de terem risco de sobrepeso ou excesso de peso, sendo que a chance de risco de sobrepeso em crianças cujas mães são obesas foi aproximadamente três vezes a chance de risco de sobrepeso em crianças cujas mães são eutróficas.

Após a seleção de todas as variáveis com valor de $\mathrm{p}<0,20$, a análise ajustada mostrou que as variáveis significativamente associadas à maior chance de risco de sobrepeso e ao excesso de peso foram, respectivamente, "idade e renda fixa" e "idade e IMC da mãe".

Conforme consta na Tabela 3, na análise ajustada a variável "renda fixa" deixou de ser significativa para o excesso de peso e o inverso foi observado para o risco de sobrepeso, ou seja, para as crianças que viviam em domicílios com renda fixa, a prevalência de risco de sobrepeso foi $97 \%$ mais elevada quando comparadas às com os domicílios sem renda fixa. 
Tabela 1

Distribuição das crianças indígenas Xukuru do Ororubá, segundo variáveis independentes e conforme com a classificação do estado nutricional pelo índice IMC-para-idade (IMC/I). Pernambuco, Brasil, 2010.

\begin{tabular}{|c|c|c|c|c|c|c|c|c|}
\hline \multirow[t]{2}{*}{ Variáveis } & \multirow[t]{2}{*}{$\mathbf{n}$} & \multicolumn{2}{|c|}{ Peso adequado } & \multicolumn{2}{|c|}{ Risco de sobrepeso } & \multicolumn{2}{|c|}{ Excesso de peso } & \multirow[t]{2}{*}{ Valor de $p$ * } \\
\hline & & $\mathbf{n}$ & $\%$ & $\mathbf{n}$ & $\%$ & n & $\%$ & \\
\hline Idade (anos) & & & & & & & & $<0,001$ \\
\hline$<2$ & 87 & 53 & 60,9 & 28 & 32,2 & 6 & 6,9 & \\
\hline$\geq 2 \mathrm{e}<5$ & 141 & 102 & 72,3 & 25 & 17,7 & 14 & 9,9 & \\
\hline$\geq 5$ e $<7$ & 83 & 75 & 90,4 & 6 & 7,2 & 2 & 2,4 & \\
\hline$\geq 7$ & 139 & 121 & 87,1 & 14 & 10,1 & 4 & 2,9 & \\
\hline Sexo & & & & & & & & 0,252 \\
\hline Masculino & 237 & 179 & 75,2 & 45 & 18,9 & 14 & 5,9 & \\
\hline Feminino & 213 & 172 & 81,1 & 28 & 13,2 & 12 & 5,7 & \\
\hline Peso ao nascer ** & & & & & & & & 0,554 \\
\hline Adequado & 163 & 131 & 80,4 & 22 & 13,5 & 10 & 6,1 & \\
\hline Inadequado & 21 & 17 & 81,0 & 2 & 9,5 & 2 & 9,5 & \\
\hline Moradores no domicílio & & & & & & & & 0,247 \\
\hline $1-4$ & 145 & 116 & 80,0 & 22 & 15,2 & 7 & 4,8 & \\
\hline $5-8$ & 179 & 129 & 72,1 & 35 & 19,6 & 15 & 8,4 & \\
\hline$>8$ & 124 & 104 & 83,9 & 16 & 12,9 & 4 & 3,2 & \\
\hline Filhos & & & & & & & & 0,897 \\
\hline$\leq 3$ & 114 & 92 & 80,7 & 16 & 14,0 & 6 & 5,3 & \\
\hline$>3$ & 188 & 147 & 78,2 & 31 & 16,5 & 10 & 5,3 & \\
\hline Idade materna (anos) & & & & & & & & 0,053 \\
\hline$<20$ & 18 & 19 & 95,0 & 1 & 5,0 & 0 & 0,0 & \\
\hline$\geq 20$ e $<35$ & 243 & 195 & 79,3 & 39 & 15,9 & 12 & 4,9 & \\
\hline$\geq 35$ & 63 & 46 & 69,7 & 11 & 16,7 & 9 & 13,6 & \\
\hline IMC da mãe $\left(\mathrm{kg} / \mathrm{m}^{2}\right)$ & & & & & & & & 0,184 \\
\hline$<25$ & 164 & 133 & 81,1 & 22 & 13,4 & 9 & 5,5 & \\
\hline$\geq 25$ e $<30$ & 92 & 74 & 77,1 & 17 & 17,7 & 5 & 5,2 & \\
\hline$\geq 30$ & 50 & 35 & 67,3 & 10 & 19,2 & 7 & 13,5 & \\
\hline Estatura materna $(\mathrm{cm})$ & & & & & & & & 0,729 \\
\hline$<150$ & 279 & 219 & 77,9 & 44 & 15,7 & 18 & 6,4 & \\
\hline$\geq 150$ & 28 & 23 & 82,1 & 5 & 17,9 & 0 & 0,0 & \\
\hline Escolaridade da mãe (anos) & & & & & & & & 0,653 \\
\hline $0-4$ & 188 & 154 & 81,9 & 25 & 13,3 & 9 & 4,8 & \\
\hline $4-9$ & 80 & 59 & 73,9 & 16 & 20,0 & 5 & 6,3 & \\
\hline $9+$ & 50 & 38 & 76,0 & 10 & 20,0 & 2 & 4,0 & \\
\hline Mãe trabalha fora de casa & & & & & & & & 0,808 \\
\hline Não & 414 & 324 & 78,3 & 66 & 15,9 & 24 & 5,8 & \\
\hline Sim & 29 & 22 & 75,9 & 6 & 20,7 & 1 & 3,4 & \\
\hline Renda per capita (salário mínimo) & & & & & & & & 0,682 \\
\hline$<1$ & 269 & 213 & 79,2 & 42 & 15,6 & 14 & 5,2 & \\
\hline$\geq 1 \mathrm{e}<2$ & 100 & 76 & 76,0 & 19 & 19,0 & 5 & 5,0 & \\
\hline$\geq 2$ e $<3$ & 57 & 43 & 75,4 & 10 & 17,5 & 4 & 7,0 & \\
\hline$\geq 3$ & 24 & 19 & 79,2 & 2 & 8,3 & 3 & 12,5 & \\
\hline ISE *** & & & & & & & & 0,604 \\
\hline Baixo & 140 & 113 & 80,7 & 17 & 12,1 & 10 & 7,1 & \\
\hline Médio & 147 & 116 & 78,9 & 25 & 17,0 & 6 & 4,1 & \\
\hline Alto & 156 & 116 & 74,4 & 30 & 19,2 & 10 & 6,4 & \\
\hline
\end{tabular}

(continua) 
Tabela 1 (continuação)

\begin{tabular}{|c|c|c|c|c|c|c|c|c|}
\hline \multirow[t]{2}{*}{ Variáveis } & \multirow[t]{2}{*}{$\mathbf{n}$} & \multicolumn{2}{|c|}{ Peso adequado } & \multicolumn{2}{|c|}{ Risco de sobrepeso } & \multicolumn{2}{|c|}{ Excesso de peso } & \multirow[t]{2}{*}{ Valor de $p$ * } \\
\hline & & $\mathbf{n}$ & $\%$ & $\mathbf{n}$ & $\%$ & $\mathbf{n}$ & $\%$ & \\
\hline Renda fixa no domicílio \# & & & & & & & & 0,147 \\
\hline Não & 216 & 177 & 81,9 & 32 & 14,8 & 7 & 3,2 & \\
\hline Sim & 231 & 172 & 74,5 & 40 & 17,3 & 19 & 8,2 & \\
\hline Domicílio \#\# & & & & & & & & 0,711 \\
\hline Tipo A & 402 & 310 & 77,1 & 68 & 16,9 & 24 & 6,0 & \\
\hline Tipo B & 45 & 38 & 84,4 & 5 & 11,1 & 2 & 4,4 & \\
\hline Saneamento \#\#\# & & & & & & & & 0,524 \\
\hline Adequado & 13 & 10 & 76,9 & 1 & 11,5 & 1 & 11,5 & \\
\hline Semiadequado & 186 & 147 & 79,0 & 28 & 15,8 & 11 & 5,9 & \\
\hline Inadequado & 249 & 189 & 75,9 & 45 & 18,1 & 15 & 6,0 & \\
\hline
\end{tabular}

IMC: índice de massa corporal; ISE: índice de status econômico.

* Teste qui-quadrado;

** Inadequado (<2.500g e $\geq 4.000 \mathrm{~g})$;

*** Obtido com base na soma em Reais de bens de consumo disponíveis no domicílio e classificado em tercis da distribuição com referência ao maior valor encontrado;

\# Presença de morador assalariado, aposentado ou pensionista;

\#\# Tipo A: paredes de alvenaria ou tijolo, ou madeira apropriada, piso de cerâmica ou cimento e telha de barro ou laje; Tipo B: Habitações com paredes de madeira aproveitada ou taipa/barro, com piso de terra ou outro material, e cobertura de lona, plástico ou outro material;

\#\#\# Adequado: escoadouros ligados à rede geral ou fossa séptica, servidos de água proveniente de rede geral de abastecimento ou rede da Fundação Nacional de Saúde (Funasa), e com destino do lixo coletado diretamente ou indiretamente pelos serviços de limpeza; Semiadequado: pelo menos, um dos serviços de abastecimento de água, esgoto ou lixo classificado como adequado; Inadequado: escoadouros ligados à fossa rudimentar, vala, açude e outro escoadouro; servidos de água proveniente de poço ou nascente, ou outra forma, com destino de lixo queimado ou enterrado, ou jogado em terreno baldio.

Tabela 2

Regressão logística multinomial bruta para o risco de sobrepeso (IMC/I $\geq 1$ escore $\mathrm{z}$ ) e excesso de peso (IMC/I $\geq 2$ escores z) de crianças indígenas Xukuru do Ororubá, segundo variáveis investigadas. Pernambuco, Brasil, 2010.

\begin{tabular}{|c|c|c|c|c|c|c|c|}
\hline \multirow[t]{2}{*}{ Variáveis } & \multirow[t]{2}{*}{$\mathbf{n}$} & \multicolumn{3}{|c|}{ Risco de sobrepeso } & \multicolumn{3}{|c|}{ Excesso de peso } \\
\hline & & RC & IC95\% & Valor de $p$ & RC & IC95\% & Valor de $p$ \\
\hline \multicolumn{8}{|l|}{ Idade (anos) } \\
\hline$<2$ & 87 & 1,00 & & & 1,00 & & \\
\hline$\geq 2 \mathrm{e}<5$ & 141 & 0,46 & $0,25-0,87$ & 0,009 & 1,21 & $0,44-3,34$ & 0,355 \\
\hline$\geq 5$ e $<7$ & 83 & 0,15 & $0,06-0,39$ & $<0,001$ & 0,24 & $0,05-1,21$ & $<0,001$ \\
\hline$\geq 7$ & 139 & 0,22 & $0,11-0,45$ & $<0,001$ & 0,29 & $0,08-1,08$ & $<0,001$ \\
\hline \multicolumn{8}{|l|}{ Sexo } \\
\hline Masculino & 237 & 1,00 & & & 1,00 & & \\
\hline Feminino & 213 & 0,65 & $0,39-1,08$ & 0,049 & 0,89 & $0,40-1,98$ & 0,390 \\
\hline \multicolumn{8}{|c|}{ Peso ao nascer * } \\
\hline Adequado & 163 & 1,00 & & & 1,00 & & \\
\hline Inadequado & 21 & 0,70 & $0,15-3,25$ & 0,325 & 1,54 & $0,31-7,63$ & 0,298 \\
\hline \multicolumn{8}{|c|}{ Moradores no domicílio } \\
\hline $1-4$ & 145 & 1,00 & & & 1,00 & & \\
\hline $5-8$ & 179 & 1,43 & $0,79-2,58$ & 0,117 & 1,93 & $0,76-4,89$ & 0,084 \\
\hline$>8$ & 124 & 0,81 & $0,40-1,63$ & 0,278 & 0,64 & $0,18-2,24$ & 0,241 \\
\hline \multicolumn{8}{|l|}{ Filhos } \\
\hline$\leq 3$ & 114 & 1,00 & & & 1,00 & & \\
\hline$>3$ & 188 & 1,21 & $0,63-2,34$ & 0,283 & 1,04 & $0,37-2,97$ & 0,469 \\
\hline
\end{tabular}

(continua) 
Tabela 2 (continuação)

\begin{tabular}{|c|c|c|c|c|c|c|c|}
\hline \multirow[t]{2}{*}{ Variáveis } & \multirow[t]{2}{*}{$\mathbf{n}$} & \multicolumn{3}{|c|}{ Risco de sobrepeso } & \multicolumn{3}{|c|}{ Excesso de peso } \\
\hline & & RC & IC95\% & Valor de $p$ & RC & IC95\% & Valor de $p$ \\
\hline \multicolumn{8}{|c|}{ Idade materna (anos) } \\
\hline$<20$ & 18 & 1,00 & & & 1,00 & & \\
\hline$\geq 20$ e $<35$ & 243 & 3,45 & $0,45-2,67$ & 0,118 & 5,93 & $0,01-7,92$ & 0,394 \\
\hline$\geq 35$ & 63 & 3,89 & $0,47-3,46$ & 0,105 & 6,67 & $0,10-10,64$ & 0,389 \\
\hline \multicolumn{8}{|l|}{ IMC da mãe (kg/m²) } \\
\hline$<25$ & 164 & 1,00 & & & 1,00 & & \\
\hline$\geq 25$ e $<30$ & 92 & 1,39 & $0,69-2,78$ & 0,177 & 1,00 & $0,32-3,09$ & 0,499 \\
\hline$\geq 30$ & 50 & 1,73 & $0,75-3,98$ & 0,100 & 2,96 & $1,03-8,49$ & 0,022 \\
\hline \multicolumn{8}{|c|}{ Estatura materna $(\mathrm{cm})$} \\
\hline$<150$ & 279 & 1,00 & & & 1,00 & & \\
\hline$\geq 150$ & 28 & 1,08 & $0,39-3,00$ & 0,440 & 0,01 & $0,01-1,78$ & 0,413 \\
\hline \multicolumn{8}{|c|}{ Escolaridade da mãe (anos) } \\
\hline $0-4$ & 188 & 1,00 & & & 1,00 & & \\
\hline $4-9$ & 80 & 1,67 & $0,83-3,35$ & 0,074 & 1,45 & $0,47-4,51$ & 0,260 \\
\hline $9+$ & 50 & 1,62 & $0,72-3,66$ & 0,123 & 0,90 & $0,19-4,34$ & 0,448 \\
\hline \multicolumn{8}{|c|}{ Mãe trabalha fora de casa } \\
\hline Não & 414 & 1,00 & & & 1,00 & & \\
\hline Sim & 29 & 1,34 & $0,52-3,43$ & 0,272 & 0,62 & $0,08-4,75$ & 0,321 \\
\hline \multicolumn{8}{|c|}{ Renda per capita (salário mínimo) } \\
\hline$<1$ & 269 & 1,00 & & & 1,00 & & \\
\hline$\geq 1 \mathrm{e}<2$ & 100 & 1,27 & $0,69-2,31$ & 0,220 & 1,01 & $0,35-2,87$ & 0,499 \\
\hline$\geq 2 \mathrm{e}<3$ & 57 & 1,18 & $0,55-2,53$ & 0,336 & 1,42 & $0,44-4,51$ & 0,279 \\
\hline$\geq 3$ & 24 & 0,53 & $0,12-2,38$ & 0,205 & 2,40 & $0,63-9,10$ & 0,099 \\
\hline \multicolumn{8}{|l|}{ ISE ** } \\
\hline Baixo & 140 & 1,00 & & & 1,00 & & \\
\hline Médio & 147 & 1,43 & $0,73-2,79$ & 0,146 & 0,58 & $0,21-1,66$ & 0,157 \\
\hline Alto & 156 & 1,72 & $0,90-3,29$ & 0,051 & 0,97 & $0,39-2,43$ & 0,478 \\
\hline \multicolumn{8}{|c|}{ Renda fixa no domicílio *** } \\
\hline Não & 216 & 1,00 & & & 1,00 & & \\
\hline Sim & 231 & 1,29 & $0,77-2,14$ & 0,167 & 2,79 & $1,15-6,81$ & 0,012 \\
\hline \multicolumn{8}{|l|}{ Domicílio \# } \\
\hline Tipo A & 402 & 1,00 & & & 1,00 & & \\
\hline Tipo B & 45 & 0,60 & $0,23-1,58$ & 0,151 & 0,68 & $0,15-2,99$ & 0,305 \\
\hline \multicolumn{8}{|l|}{ Saneamento \#\# } \\
\hline Adequado & 13 & 1,00 & & & 1,00 & & \\
\hline Semiadequado & 186 & 2,44 & $0,01-1,65$ & 0,406 & 1,23 & $0,01-3,49$ & 0,424 \\
\hline Inadequado & 249 & 3,05 & $0,02-2,07$ & 0,403 & 1,29 & $0,30-3,69$ & 0,423 \\
\hline
\end{tabular}

IC95\%: intervalo de 95\% de confiança; IMC: índice de massa corporal; IMC/I: índice IMC-para-idade; ISE: índice de status econômico; RC: razão de chances.

* Inadequado (<2.500g e $\geq 4.000 \mathrm{~g})$;

** Obtido com base na soma em Reais de bens de consumo disponíveis no domicílio;

*** Presença de morador assalariado, aposentado ou pensionista;

\# Tipo A: paredes de alvenaria ou tijolo, ou madeira apropriada, piso de cerâmica ou cimento e telha de barro ou laje; Tipo B: habitações com paredes de madeira aproveitada ou taipa/barro, com piso de terra ou outro material, e cobertura de lona, plástico ou outro material;

\#\# Adequado: escoadouros ligados à rede geral ou fossa séptica, servidos de água proveniente de rede geral de abastecimento ou rede da Fundação Nacional de Saúde (Funasa), e com destino do lixo coletado diretamente ou indiretamente pelos serviços de limpeza; Semiadequado: pelo menos, um dos serviços de abastecimento de água, esgoto ou lixo classificado como adequado; Inadequado: escoadouros ligados à fossa rudimentar, vala, açude e outro escoadouro; servidos de água proveniente de poço ou nascente, ou outra forma, com destino de lixo queimado ou enterrado, ou jogado em terreno baldio. 
Tabela 3

Regressão logística multinomial ajustada para o risco de sobrepeso (IMC/I $\geq 1$ e $>2$ escores z) e excesso de peso (IMC/I $\geq$ 2 escores z) de crianças indígenas Xukuru do Ororubá. Pernambuco, Brasil, 2010.

\begin{tabular}{|c|c|c|c|c|c|c|}
\hline \multirow[t]{2}{*}{ Variáveis } & \multicolumn{3}{|c|}{ Risco de sobrepeso } & \multicolumn{3}{|c|}{ Excesso de peso } \\
\hline & RC & IC95\% & Valor de $p$ & RC & IC95\% & Valor de $p$ \\
\hline \multicolumn{7}{|l|}{ Idade (anos) } \\
\hline$<2$ & 1,00 & & & 1,00 & & \\
\hline$\geq 2 \mathrm{e}<5$ & 0,37 & $0,17-0,83$ & 0,008 & 0,64 & $0,20-2,01$ & 0,222 \\
\hline$\geq 5$ e $<7$ & 0,13 & $0,04-0,40$ & $<0,001$ & 0,15 & $0,03-0,85$ & 0,016 \\
\hline$\geq 7$ & 0,12 & $0,05-0,33$ & $<0,001$ & 0,18 & $0,04-0,76$ & $<0,001$ \\
\hline \multicolumn{7}{|l|}{ Sexo } \\
\hline Masculino & 1,00 & & & 1,00 & & \\
\hline Feminino & 0,58 & $0,30-1,14$ & 0,050 & 0,63 & $0,25-1,63$ & 0,172 \\
\hline \multicolumn{7}{|c|}{ IMC da mãe (kg/m²) } \\
\hline$<25$ & 1,00 & & & 1,00 & & \\
\hline$\geq 25$ e $<30$ & 1,55 & $0,73-3,27$ & 0,127 & 1,21 & $0,38-3,87$ & 0,375 \\
\hline$\geq 30$ & 1,95 & $0,80-4,76$ & 0,072 & 3,39 & $1,12-10,27$ & 0,016 \\
\hline \multicolumn{7}{|c|}{ Renda fixa no domicílio * } \\
\hline Não & 1,00 & & & & & \\
\hline Sim & 1,97 & $1,01-3,88$ & 0,026 & 2,58 & $0,96-6,94$ & 0,031 \\
\hline
\end{tabular}

IC95\%: intervalo de 95\% de confiança; IMC: índice de massa corporal; IMC/I: índice IMC-para-idade; RC: razão de chances.

* Presença de morador assalariado, aposentado ou pensionista.

A associação do IMC materno com o excesso de peso infantil permaneceu relevante na análise ajustada. A prevalência do agravo em filhos de mães obesas foi $239 \%$ maior quando comparadas aos filhos de mães eutróficas.

A análise ajustada apontou também que em relação à idade das crianças, para ambos os desfechos, o menor grupamento de idade considerado ( $<2$ anos) implicava o maior risco. A prevalência de risco de sobrepeso foi significativamente decrescente com o aumento da idade a partir dos 2 anos, sendo nos maiores de 7 anos $88 \%$ menor, quando comparada com os menores de 2 anos. Já para o excesso de peso, essa relação foi observada apenas para os maiores de 5 anos, com prevalências $85 \%$ e $82 \%$ menores para crianças com idades entre 5 e 7 anos e maiores de 7 anos, respectivamente, quando comparadas com as menores de 2 anos.

\section{Discussão}

O perfil antropométrico das crianças Xukuru do Orurubá distingue-se de estudos com outras comunidades indígenas no Brasil, onde as prevalências de sobrepeso e obesidade são praticamente nulas no segmento infantil 11,12,13. O déficit de crescimento e baixo peso são considerados agravos expressivos entre as crianças indígenas do Brasil 20, não sendo os principais problemas nutricionais infantis nos Xukuru do Orurubá.

As frequências de excesso de peso em crianças indígenas Xukuru do Orurubá aproximam-se das encontradas em crianças brasileiras não indígenas menores de 5 anos 21. Os resultados da III Pesquisa Estadual de Saúde e Nutrição de Pernambuco revelaram uma prevalência de excesso de peso para crianças não indígenas entre 2 e 5 anos de 8,1\%. Para as crianças que viviam na zona rural, o valor encontrado foi de 6,8\% 22. E um dos últimos inquéritos nacionais que avaliaram o estado nutricional de crianças identificou 6,2\% de excesso de peso no Nordeste 23 . Ambos os resultados são semelhantes aos achados do presente trabalho. Porém, outros estudos em diferentes regiões do Nordeste, especialmente na região do semiárido, têm ressaltado prevalências ainda maiores de sobrepeso e obesidade em crianças 24,25 . 
O segmento infantil, assim como outros considerados vulneráveis frente ao contexto de pobreza e exclusão, tem sido fortemente relacionado à obesidade por alguns autores 26,27. Assim, problemas relacionados ao excesso de peso são cada vez mais evidenciados em regiões com os piores indicadores socioeconômicos 27 . Historicamente, a macrorregião Nordeste é uma das regiões geográficas do Brasil com as piores rendas e maiores desigualdades em saúde, e os indígenas são reconhecidos como um dos grupos populacionais mais vulneráveis do ponto de vista social e econômico no Nordeste e no Brasil 28.

A obesidade é considerada um agravo nutricional importante entre adultos indígenas do Brasil, não raro sendo reportadas frequências de sobrepeso e/ou obesidade na ordem de 70-80\% 15,29,30. Entre adolescentes, os poucos estudos com indígenas do país sinalizaram também prevalências elevadas de excesso de peso e percentual de gordura corporal 31,32.

Em crianças indígenas na América do Norte e na América do Sul há evidências na literatura do aumento nas prevalências de obesidade 33,34. Porém, as poucas investigações que destacam a presença de sobrepeso ou obesidade em crianças indígenas no Brasil são entre indivíduos das macrorregiões Sul 35,36,37 e Sudeste 38 do país. Um deles, incluindo crianças Kaingáng < 5 anos de idade, sinalizou a presença de 6,4\% de crianças com sobrepeso, de acordo com o IMC/I e o padrão de referência da OMS 36. Já em outro estudo com o mesmo grupo étnico, mas com crianças de outra Terra Indígena, o valor de excesso de peso foi aproximadamente 10\%, de acordo com o índice peso-para-altura e segundo a população referência do Centro Nacional de Estatísticas de Saúde (NCHS) dos Estados Unidos 37.

A classificação de sobrepeso e obesidade para crianças foi modificada desde o lançamento das curvas de crescimento e padrão da OMS em 2006 e 2007. A partir de então, a proposta para a classificação da obesidade infantil envolve o uso do IMC [peso $(\mathrm{kg}) /$ altura $(\mathrm{m})^{2}$ ] em relação à idade (IMC/I), com base em uma referência internacionalmente preconizada 18,19 . Os índices anteriormente usados para classificar o excesso de peso em crianças não levavam em consideração a idade, o peso e a estatura conjuntamente, sofrendo inúmeros vieses, além de apresentar problemas metodológicos na seleção da amostra da população de referência 18,19,39.

Ainda que para a avaliação da obesidade infantil diferentes classificações e referências tenham sido propostas, o IMC/I tem sido o mais utilizado e o internacionalmente preconizado para fins de comparação e classificação de sobrepeso e obesidade em crianças referência 18,19,39. No Brasil, a recomendação do Ministério da Saúde também é a utilização do IMC/I, seguindo os pontos de corte sugeridos pela OMS 40. Contudo, estudos recentes ainda usam outros índices para classificar o excesso de peso infantil, dificultando a comparação. É o caso da investigação de Pereira et al. 9, realizada com crianças indígenas Karapotó em Alagoas, que encontrou 23,8\% de risco de sobrepeso e sobrepeso pelo índice peso-para-estatura.

Nas crianças Xukuru do Orurubá, quanto maior a idade da criança, menor a chance destas terem excesso de peso e risco de sobrepeso, consistente com outros estudos 41,42. Assim como já foi demonstrado em um trabalho com indígenas da Austrália, onde a prevalência de sobrepeso diminuía conforme aumentava a idade 43 . Contudo, os achados da relação da idade durante a infância com o excesso de peso são contraditórios na literatura. O oposto foi demonstrado em investigação com crianças $<5$ anos do semiárido do Brasil, onde quanto maior a idade, maiores as chances de sobrepeso 24.

A prevalência da obesidade em crianças reflete em grande parte as modificações mais recentes nos padrões alimentares e inatividade física. Os processos de urbanização, globalização, aumento da renda, aumento de bens de consumo duráveis, entre outros, contribuíram para um conjunto de modificações no estilo de vida que impactaram diretamente no aumento do sobrepeso e obesidade em crianças 1,44, incluindo as indígenas de outras partes do mundo 45 . Portanto, diversos fatores socioeconômicos, ambientais e demográficos têm sido sinalizados como importantes preditores da obesidade infantil 2,3,46, destacando também a alta relação do meio familiar com a obesidade da criança 3 .

A obesidade dos pais é um determinante consolidado da obesidade infantil. Diferentes investigações têm apontado uma forte relação do IMC da mãe com o estado nutricional da criança 2,4,41, assim como os achados do presente estudo. Os mecanismos da influência do estado nutricional da mãe na programação metabólica ou genética da adiposidade dos filhos ainda estão em debate. Alguns trabalhos demonstraram que a toxidade causada pelo aumento nos níveis de lipídios no organismo pode alterar o metabolismo fetal, mas não está claro como este processo pode influenciar no metabolismo da criança ao longo da infância 47. 
De fato, grande parte da variabilidade do IMC pode ser atribuída a interações genéticas, ambientais e comportamentais, desde o período intrauterino 2. O ambiente é considerado particularmente importante, especialmente o familiar, pois os pais (ou cuidadores) exercem considerável influência sobre o ambiente alimentar, social e econômico das crianças nos primeiros anos de vida, que são fatores determinantes para a obesidade infantil 3,44. Além disso, pais e mães obesos são mais propensos a criar um estilo de vida e ambiente mais obesogênico, aumentando as chances de excesso de peso na criança 42 . Os adultos Xukuru, investigados no mesmo âmbito da coleta de dados que resultou no presente estudo, apresentaram prevalências bastante elevadas de excesso de peso e obesidade 15. Considerando o modo de seleção amostral realizada, em que foram incluídos os moradores das residências sorteadas, presume-se que as crianças Xukuru também estejam expostas ao mesmo ambiente obesogênico.

Nesse contexto, as condições socioeconômicas também são muito importantes para o aumento de chances de excesso de peso em crianças. Estudos sugerem que o aumento dos níveis de obesidade coincide com a melhoria da economia e rendimentos do país, como é o caso do Brasil 2. Blüher 2 ainda sinaliza que no país, assim como em outros países de menor nível socioeconômico, a prevalência de obesidade geralmente aumenta primeiro em indivíduos com maior estrato socioeconômico nas áreas urbanas e, em seguida, em grupos de menor estrato socioeconômico, predominantemente nas zonas rurais. Trabalhos com crianças indígenas australianas também têm sinalizado a associação significativa do nível socioeconômico com o excesso de peso 45,48, inclusive indicando que o nível socioeconômico tem efeito diferente no IMC de crianças indígenas quando comparadas a crianças não indígenas 49 .

Um rendimento mensal fixo, por sua vez, pode contribuir para uma exposição a alimentos e ambientes considerados mais obesogênicos 2. Em um estudo multinacional com crianças escolares, conduzido em 12 centros urbanos de países diferentes, incluindo o Brasil, os escores do IMC/I aumentaram linearmente conforme uma maior renda em países com os mais baixos níveis de desenvolvimento. Esse resultado era o inverso em países considerados de estratos socioeconômicos superiores 46 .

É importante ressaltar que os poucos estudos que avaliaram saúde e estado nutricional dos indígenas que vivem na macrorregião Nordeste do país sinalizaram para altas prevalências de sobrepeso, obesidade e outras doenças crônicas $15,28,50$. Os fatores socioeconômicos têm sido importantes variáveis associadas a esse cenário ${ }^{15}$.

Outros aspectos relacionados ao desenvolvimento do excesso de peso em crianças não foram alvos de investigação neste trabalho, limitando algumas relações que poderiam ser estabelecidas, como a investigação do consumo alimentar principalmente quanto ao aleitamento materno, alimentação complementar e consumo de alimentos ultraprocessados. A decisão metodológica pela utilização do IMC/I limitou algumas comparações com estudos que optaram pelo índice peso-para-altura.

\section{Considerações finais}

O Brasil precisa de políticas de investimento para identificar e minimizar os determinantes do excesso de peso em crianças indígenas e não indígenas. Na Austrália, um estudo de base populacional evidenciou que a escolaridade materna e a estabilidade habitacional afetam o consumo de refrigerantes por crianças indígenas que vivem em bairros vizinhos, e tal fato foi considerado na definição de políticas públicas 51 .

Os resultados aqui debatidos unem-se a outros que retratam a situação paradoxal a que estão expostos muitos povos indígenas no Brasil. São diversas realidades sanitárias refletidas em indicadores de saúde invariavelmente piores do que os observados em contextos não indígenas, e acompanhados por outros extratos populacionais pauperizados e altamente vulneráveis da sociedade brasileira. Desse modo, ao menos no que compete diretamente ao setor saúde, é imprescindível a discussão de uma nova política de saúde indígena que considere o cenário nutricional desenhado aqui. As abordagens individualistas da medicina ocidental no tocante ao manejo do excesso de peso para populações indígenas, quer sejam adultos ou crianças, devem ser estratégicas alinhadas culturalmente ao modo de ser indígena. 


\section{Colaboradores}

T. R. Fávaro contribuiu com a concepção do projeto, análise e discussão dos resultados, redação, revisão crítica relevante do conteúdo intelectual e revisão da versão final. A. A. Ferreira contribuiu com a análise e discussão dos resultados, redação, revisão crítica relevante do conteúdo intelectual e revisão da versão final. G. M. Cunha contribuiu com a concepção do projeto, análise e interpretação dos dados e com a aprovação da versão final. C. E. A. Coimbra Jr. contribuiu com a concepção do projeto, revisão crítica relevante do conteúdo intelectual e revisão da versão final.

\section{Informações adicionais}

ORCID: Thatiana Regina Fávaro (0000-0001-72753245); Aline Alves Ferreira (0000-0001-50813462); Geraldo Marcelo da Cunha (0000-00017128-933X); Carlos E. A. Coimbra Jr. (0000-00034085-1080).

\section{Referências}

1. World Health Organization. Report of the Commission on Ending Childhood Obesity. Implementation Plan: executive summary. Geneva: World Health Organization; 2017. (WHO/NMH/PND/ECHO/17.1).

2. Blüher M. Obesity: global epidemiology and pathogenesis. Nat Rev Endocrinol 2019; 15:288-98.

3. Vos MB, Welsh J. Childhood obesity: update on predisposing factors and prevention strategies. Curr Gastroenterol Rep 2010; 12:280-7.

4. Porter RM, Tindall A, Gaffka BJ, Kirk S, Santos M, Abraham-Pratt I, et al. A review of modifiable risk factors for severe obesity in children ages 5 and under. Child Obes 2018; 14:468-76.

5. Conde WL, Monteiro CA. Nutrition transition and double burden of undernutrition and excess of weight in Brazil. Am J Clin Nutr 2014; 100:1617S-22S.

6. Barreto CTG, Cardoso AM, Coimbra Jr. CEA. Estado nutricional de crianças indígenas Guarani nos estados do Rio de Janeiro e São Paulo, Brasil. Cad Saúde Pública 2014; 30:657-62.

7. Ferreira AA, Santos RV, Souza JAM, Welch JR, Coimbra Jr. CEA. Anemia e níveis de hemoglobina em crianças indígenas Xavante, Brasil Central. Rev Bras Epidemiol 2017; 20:102-14.

\section{Agradecimentos}

Agradecemos à população Xukuru do Ororubá pela recepção à equipe de pesquisa e aos pesquisadores do Instituto Aggeu Magalhães, Fundação Oswaldo Cruz (IAM/Fiocruz), em especial ao pesquisador André Monteiro Costa. Esta pesquisa é derivada de projetos financiados pela FACEPE (processo no $\mathrm{APQ}-1351-4.00 / 08)$ e CNPq (processo no 475681/2008), e foi realizada durante a vigência de bolsa de doutorado (CNPq) e pós-doutorado (Convênio Fiocruz/Capes - Brasil sem Miséria) concedida à primeira autora.
8. Pantoja LN, Orellana JDY, Leite MS, Basta PC. Cobertura do Sistema de Vigilância Alimentar e Nutricional Indígena (SISVAN-I) e prevalência de desvios nutricionais em crianças Yanomami menores de 60 meses, Amazônia, Brasil. Rev Bras Saúde Mater Infant 2014; 14:53-63.

9. Pereira JF, Oliveira MAA, Oliveira JS. Anemia em crianças indígenas da etnia Karapotó. Rev Bras Saúde Mater Infant 2012; 12:375-82.

10. Associação Brasileira de Saúde Coletiva. Inquérito Nacional de Saúde e Nutrição dos Povos Indígenas. Relatório final. http://ecos -redenutri.bvs.br/tiki-download_file.php?fi leId=1284 (acessado em 12/Mar/2019).

11. Escobar AL, Santos RV, Coimbra Jr. CEA. Avaliação nutricional de crianças indígenas Pakaanóva (Wari'), Rondônia, Brasil. Rev Bras Saúde Mater Infant 2003; 3:457-61.

12. Ferreira AA, Welch JR, Santos RV, Gugelmin SA, Coimbra Jr. CEA. Nutritional status and growth of indigenous Xavante children, Central Brazil. Nutr J 2012; 11:1-9.

13. Mondini L, Canó EN, Fagundes U, Lima EES, Rodrigues D, Baruzzi RG. Condições de nutrição em crianças Kamaiurá - povo indígena do Alto Xingu, Brasil Central. Rev Bras Epidemiol 2007; 10:39-47. 
14. Mauricio HA, Moreira RS. Oral health status of the ethnic group Xukuru from Ororubá: multilevel analysis. Rev Bras Epidemiol 2014; 17:787-800

15. Fávaro TR, Santos RV, Cunha GM, Leite IC, Coimbra Jr. CEA. Obesidade e excesso de peso em adultos indígenas Xukuru do Ororubá, Pernambuco, Brasil: magnitude, fatores socioeconômicos e demográficos associados. Cad Saúde Pública 2015; 31:1685-97.

16. Teixeira P, Costa AM. Xukuru de Ororubá: introdução à demografia de um povo indígena. In: Anais do XVIII Encontro Nacional de Estudos Populacionais. http://www.abep. org.br/publicacoes/index.php/anais/article/ view/1913/1871.

17. Lohman TG, Roche AF, Martorell R. Anthropometric standardization reference manual. Champaign: Human Kinetics; 1988.

18. Onis M, Onyango AW, Borghi E, Siyam A, Nishida C, Siekmann J. Development of a WHO growth reference for school-aged children and adolescents. Bull World Health Organ 2007; 85:660-7.

19. World Health Organization. Length/heightfor-age, weight-for-age, weight-for-length, weight-forheight and body mass index-forage: methods and development. Geneva: World Health Organization; 2006.

20. Horta BL, Santos RV, Welch JR, Cardoso AM, dos Santos JV, Assis AM, et al. Nutritional status of indigenous children: findings from the First National Survey of Indigenous People's Health and Nutrition in Brazil. Int J Equity Health 2013; 12:23.

21. Instituto Brasileiro de Geografia e Estatística. Pesquisa de Orçamentos Familiares 20082009 - antropometria e estado nutricional de crianças, adolescentes e adultos no Brasil. Rio de Janeiro: Instituto Brasileiro de Geografia e Estatística; 2010.

22. Menezes RCE, Lira PIC, Leal VS, Oliveira JS, Santana SCS, Sequeira LAS, et al. Determinantes do déficit estatural em menores de cinco anos no Estado de Pernambuco. Rev Saúde Pública $2011 ; 45: 1079-87$.

23. Meller FO, Araújo CLP, Madruga SW. Fatores associados ao excesso de peso em crianças brasileiras menores de cinco anos. Ciênc Saúde Colet 2014; 19:943-55.

24. Oppitz IN, Cesar JA, Neumann NA. Excesso de peso entre menores de cinco anos em municípios do semiárido. Rev Bras Epidemiol 2014; 17:860-72.

25. Ramos CV, Dumith SC, César JA. Prevalence and factors associated with stunting and excess weight in children aged 0-5 years from the Brazilian semi-arid region. J Pediatr (Rio J.) 2015; 91:175-82.

26. Carpenter DO, Sly PD. Risk factors for poor health in indigenous communities. Rev Environ Health 2018; 33:319.

27. Ferreira VA, Magalhães R. Obesidade entre os pobres no Brasil: a vulnerabilidade feminina. Ciênc Saúde Colet 2011; 16:2279-87.
28. Coimbra Jr. CEA, Santos RV, Welch JR, Cardoso AM, de Souza MC, Garnelo L, et al. The First National Survey of Indigenous People's Health and Nutrition in Brazil: rationale, methodology, and overview of results. BMC Public Health 2013; 13:52.

29. Welch JR, Ferreira AA, Santos RV, Gugelmin SA, Werneck G, Coimbra Jr. CEA. Nutrition transition, socioeconomic differentiation, and gender among adult Xavante Indians, Brazilian Amazon. Hum Ecol 2009; 37:13-26.

30. Lourenço AEP, Santos RV, Orellana JDY, Coimbra Jr. CEA. Nutrition transition in Amazonia: obesity and socioeconomic change in the Suruí Indians from Brazil. Am J Hum Biol 2008; 20:564-71.

31. Barufaldi LA, Conde WL, Schuch I, Duncan $\mathrm{BB}$, Castro TG. Bioelectrical impedance values among indigenous children and adolescents in Rio Grande do Sul, Brazil. Rev Panam Salud Pública 2011; 30:39-45.

32. Castro TG, Barufaldi LA, Schluessel MM, Conde WL, Leite MS, Schuch I. Waist circumference and waist circumference to height ratios of Kaingang indigenous adolescents from the State of Rio Grande do Sul, Brazil. Cad Saúde Pública 2012; 28:2053-62.

33. Schell LM, Gallo MV. Overweight and obesity among North American Indian infants, children, and youth. Am J Hum Biol 2012; 24:30213.

34. Bustos P, Munõz S, Vargas C, Amigo H. Poverty and indigenous origin as risk factors of nutritional problems among children who enroll in school. Salud Pública Méx 2018; 51:187-93.

35. Castro TG, Schuch I, Conde WL, Veiga J, Leite MS, Dutra CLC, et al. Estado nutricional dos indígenas Kaingáng matriculados em escolas indígenas do Estado do Rio Grande do Sul, Brasil. Cad Saúde Pública 2010; 26:1766-76.

36. Kühl AM, Corso ACT, Leite MS, Bastos JL. Perfi 1 nutricional e fatores associados à ocorrência de desnutrição entre crianças indígenas Kaingáng da Terra Indígena de Mangueirinha, Paraná, Brasil. Cad Saúde Pública 2009; 25:409-20.

37. Menegolla IA, Drachler ML, Rodrigues IH, Schwingel LR, Scapinello E, Pedroso MB, et al. Estado nutricional e fatores associados à estatura de crianças da Terra Indígena Guarita, Sul do Brasil. Cad Saúde Pública 2006; 22:395406.

38. Santos AP, Mazzeti CMS, Franco MCP, Santos NLGO, Conde WL, Leite MS, et al. Estado nutricional e condições ambientais e de saúde de crianças Pataxó, Minas Gerais, Brasil. Cad Saúde Pública 2018; 34:e00165817.

39. de Onis M, Lobstein T. Defining obesity risk status in the general childhood population: which cut-offs should we use? Int J Pediatr Obes 2010; 5:458-60.

40. Ministério da Saúde. Orientações para coleta e análise de dados antropométricos em serviços de saúde: norma técnica do Sistema de Vigilância Alimentar e Nutricional - SISVAN. Brasília: Ministério da Saúde; 2011. 
41. Linabery AM, Nahhas RW, Johnson W, Choh AC, Towne B, Odegaard AO, et al. Stronger influence of maternal than paternal obesity on infant and early childhood body mass index: the Fels Longitudinal Study. Pediatr Obes 2013; 8:159-69.

42. Olson CM, Demment MM, Carling SJ, Strawderman MS. Associations between mothers' and their children's weights at 4 years of age. Child Obes 2010; 6:201-7.

43. Schultz R. Prevalences of overweight and obesity among children in remote Aboriginal communities in central Australia. Rural Remote Health 2012; 12:1872.

44. Swinburn BA, Sacks G, Hall KD, Finegood DT, Moodie ML, Gortmaker SL. The global obesity pandemic: shaped by global drivers and local environments. Lancet 2011; 378:804-14.

45. Spurrier NJ, Volkmer RE, Abdallah CA, Chong A. South Australian four-year-old Aboriginal children: residence and socioeconomic status influence weight. Aust N Z J Public Health 2012; 36:285-90.

46. Broyles ST, Denstel KD, Church TS, Chaput JP, Fogelholm M, Hu G, et al. The epidemiological transition and the global childhood obesity epidemic. Int J Obes Suppl 2015; 5 Suppl 2:S3-8.

47. Andres A, Hull HR, Shankar K, Casey PH, Cleves MA, Badger TM. Longitudinal body composition of children born to mothers with normal weight, overweight, and obesity. Obesity (Silver Spring) 2015; 23:1252-8.
48. Wake M, Hardy P, Canterford L, Sawyer M, Carlin JB. Overweight, obesity and girth of Australian preschoolers: prevalence and socioeconomic correlates. Int J Obes 2006; 31:104451.

49. Kim S, Macaskill P, Baur L, Hodson EM, Daylight J, Williams $\mathrm{R}$, et al. The differential effect of socio-economic status, birth weight and gender on body mass index in Australian Aboriginal children. Int J Obes 2016; 40:1089-95.

50. Barbosa JMV. Prevalência e fatores associados à pressão arterial elevada no povo indígena Xukuru do Ororubá, Pesqueira - PE, 2010 [Dissertação de Mestrado]. Recife: Instituto Aggeu Magalhães, Fundação Oswaldo Cruz; 2013.

51. Laws R, Campbell KJ, van der Pligt P, Russell G, Ball K, Lynch J, et al. The impact of interventions to prevent obesity or improve obesity related behaviours in children ( $0-5$ years) from socioeconomically disadvantaged and/or indigenous families: a systematic review. BMC Public Health 2014; 14:779. 


\section{Abstract}

The few studies on health and nutrition in indigenous peoples in Northeast Brazil point to some differences with indigenous peoples in the North and Central of the country. This study estimated the prevalence rates and risk of overweight and excess weight in Xukuru children in the village of Ororubá, Pernambuco State, and assessed the socioeconomic and demographic factors potentially associated with these conditions. This cross-sectional study analyzed the associations between adequate weight, excess weight (overweight and obesity), and risk of overweight according to the indices and cutoff points of the World Health Organization for children and the explanatory variables, using multinomial logistic regression. Prevalence of excess weight was $7.7 \%$ and risk of overweight was $24.2 \%$. The odds of risk of overweight and excess weight were higher in children $<2$ years. Children of obese mothers showed higher odds of excess weight. Prevalence of risk of overweight was 97\% higher when compared to households with fixed income. The findings suggest that the Xukuru are experiencing an accelerated nutritional transition, with a paradoxical situation to which other indigenous peoples in Brazil are also exposed.

Health of Indigenous Peoples; South American Indians; Nutritional Status; Pediatric Obesity

\section{Resumen}

Los escasos estudios que abordan el tema de salud y nutrición en indígenas de la macrorregión Nordeste de Brasil mostraron situaciones diferenciadas en algunos aspectos, cuando se comparan principalmente con la realidad de los indígenas del Norte y Centro-oeste. El objetivo de este trabajo fue estimar la magnitud de las prevalencias y riesgo de sobrepeso, así como de exceso de peso en niños menores de diez años de la etnia Xukuru do Ororubá, estado de Pernambuco, y evaluar los factores socioeconómicos y demográficos potencialmente asociados a estos problemas. Se trata de un estudio transversal, en el que se realizó un análisis de la asociación entre las variables de desenlace, peso adecuado, exceso de peso (sobrepeso y obesidad) y riesgo de sobrepeso, conforme los índices y puntos de corte de la Organización Mundial de la Salud para niños, y las variables explicativas, utilizando una regresión logística multinomial. La prevalencia de exceso de peso fue de un 7,7\% y la de riesgo de sobrepeso de $24,2 \%$. Las oportunidades de riesgo de sobrepeso y de exceso de peso son mayores en niños $<2$ años, asimismo, hijos de madres obesas presentan una mayor oportunidad de exceso de peso. La prevalencia de riesgo de sobrepeso fue un $97 \%$ mayor cuando se compara con domicilios sin ingresos fijos. Los resultados sugieren que los Xukuru están atravesando un acelerado proceso de transición nutricional, con una situación paradójica a la que otros pueblos indígenas en Brasil están expuestos.

Salud de Poblaciones Indígenas; Indios

Sudamericanos; Estado Nutricional;

Obesidad Pediátrica
Recebido em 20/Mar/2019

Versão final reapresentada em 10/Mai/2019

Aprovado em 16/Mai/2019 